\title{
On the Sum of Gamma-Gamma Variates with Application to the Fast Outage Probability Evaluation Over Fading Channels
}

\author{
Chaouki Ben Issaid, Nadhir Ben Rached, Abla Kammoun, Mohamed-Slim
}

Alouini, and Raul Tempone

King Abdullah University of Science and Technology (KAUST),

Computer, Electrical, and Mathematical Sciences and Engineering (CEMSE)

Division,

Thuwal, Makkah Province, Saudi Arabia

Email: \{chaouki.benissaid, nadhir.benrached, abla.kammoun, slim.alouini, raul.tempone\}@kaust.edu.sa

\begin{abstract}
The Gamma-Gamma distribution has recently emerged in a number of applications ranging from modeling scattering and reverbation in sonar and radar systems to modeling atmospheric turbulence in wireless optical channels. In this respect, assessing the outage probability achieved by some diversity techniques over this kind of channels is of major practical importance. In many circumstances, this is intimately related to the difficult question of analyzing the statistics of a sum of Gamma-Gamma random variables. Answering this question is not a simple matter. This is essentially because outage probabilities encountered in practice are often very small, and hence the use of classical Monte Carlo methods is not a reasonable choice. This lies behind the main motivation of the present work. In particular, this paper proposes a new approach to estimate the left tail of the sum of independent and identically distributed Gamma-Gamma variates. More specifically, we propose a mean-shift importance sampling scheme that efficiently evaluates the outage probability of $L$-branch maximum ratio combining diversity receivers
\end{abstract}


over Gamma-Gamma fading channels. The proposed estimator satisfies the well-known bounded relative error criterion. We show the accuracy and the efficiency of our approach compared to naive Monte Carlo via some selected numerical simulations.

\section{Index Terms}

Gamma-Gamma, generalized-K, importance sampling, Monte Carlo, bounded relative error, outage probability, maximum ratio combining.

\section{INTRODUCTION}

The Gamma-Gamma distribution has recently emerged in many communication systems. In radar and sonar systems, it has been used to model scattering [1] and reverbation [2]. It has been broadly accepted as an appropriate model for both line-of sight and non-line-of sight wireless radio-frequency channels [3, 4]. Lately, it started to gain popularity in modeling atmospheric turbulence in wireless optical channels $[5,6]$. It was shown that this probability density function (PDF) models accurately the atmospheric turbulence in free-space optics (FSO) over a wide range being a good fit for both weak and strong turbulence [7, 8]. A concise review of the use of Gamma-Gamma to model the statistics of the signal fading channels can be found in [7].

The statistics of the sum of Gamma-Gamma RVs is needed, for instance, when investigating the efficiency of certain diversity techniques, e.g. maximum ratio combining (MRC) [9]. There have been few attempts to derive analytically the sum of independent and identically distributed (i.i.d) Gamma-Gamma variates. The first attempt was in [10] where the authors presented the PDF of the sum as a nested series when the fading parameters are integers. For the non-integer case, an infinite series representation was derived in [11] using the generalized power series representation of the modified Bessel function of the second kind. The analytical complications and the computational burden of deriving the exact PDF of the sum led many authors to turn their attention to look for an approximate distribution. In [12], the authors used a single Gamma PDF as an approximate distribution of the generalized-K PDF. By matching the first moment and the amount of fading of the instantaneous power in both cases, the parameters of the Gamma PDF were derived. A similar approach was introduced in [13] where a Gamma-Gamma PDF is used to approximate the distribution of the sum. Matching the moment generating functions (MGFs) of the generalized-K and the Gamma-Gamma PDFs led to determine the expression of 
the parameters of the approximate PDF. In [14], the distribution of the sum was approximated by a single Gamma-Gamma distribution. A more general distribution, $\alpha-\mu$, was used in [15], to fit the distribution of the sum. In this work, Peppas used the moment matching technique, and more specifically, the first, the second, and the fourth moments were used to determine the parameters of the $\alpha-\mu$ distribution. However, it is well-known that an approximation has the risk to lead to an inaccurate representation of the distribution of the sum of the RVs for a certain set of parameters. Indeed, although it often simplifies the analysis without producing large errors, it may be that such an approximation is sensitive to the parameters of the problem. For the sake of illustration, the authors in [13] have shown a clear discrepancy between simulation and the approximate approaches presented in $[12,14,15]$. To overcome this problem, one can resort to a numerical simulation approach, e.g. Monte Carlo (MC) method, which can provide more accurate results.

In this work, we are interested in estimating the probability of rare events (e.g. of the order of $10^{-9}$ ), for which the MC method is inaccurate if the sample size is not large enough [16]. In fact, a naive MC estimator, based on a reasonably limited number of simulation runs, may provide irrelevant results. For example, suppose we want to compute a probability $p=10^{-8}$ and we decided to use only $10^{6}$ samples. With a probability greater than 0.99 , the result will be equal to zero providing irrelevant information on the value of $p$ and the $99 \%$ confidence interval in this case is $\left[0,4.6 \times 10^{-6}\right]$, thus a grossly erroneous estimation [17]. In order to obtain reasonably accurate results in the rare events setting, one can use variance reduction techniques, for instance the importance sampling (IS) method [18]. IS introduces a new distribution, often called biased PDF, that "encourages" the sampling of values from the importance region. The fundamental point in the implementation of IS is the choice of the biased distribution. In fact, the advantage can then be a tremendous computational time savings while the disadvantage, in the case of a poor choice of biased distribution, is an estimator with a larger variance than a MC estimator. This is the reason why an IS estimator needs to satisfy certain criteria to guarantee the efficiency of the method.

In this paper, we propose an efficient mean-shift IS scheme for the estimation of the outage probability of L-branch MRC diversity receivers over i.i.d Gamma-Gamma fading channels. More precisely, we select the biased PDF as a Gamma-Gamma distribution with shifted mean. The main result of our work is that the proposed IS approach possesses the well-desired bounded relative 
error property, which implies that the number of samples required to meet a fixed accuracy requirement remains bounded independently of how small is the outage probability. Due to page limitation, we are not including all the details in the present conference version. Readers are referred to our extended journal version [19] where detailed proofs are presented. Moreover, we enlarge the scope of the applicability of our approach by proving its efficiency in the i.n.i.d case as well. It is important to note that, to the best of the authors knowledge, the popularity of the IS method to estimate the outage probability with diversity techniques among researchers in wireless communications is quite limited. Recently, the authors in [20] have addressed this problem using two unified IS schemes when the variates are assumed to be i.i.d. However, sampling according to their biased PDF is not straightforward in the Gamma-Gamma fading model scenario.

The remainder of this paper is organized as follows. We start by describing the system model in Section II. We then provide in Section III a brief description of the fundamental concepts of IS method. In section IV, we present our mean-shift approach to estimate the outage probability in our particular set-up. We also discuss the efficiency of the proposed method. In Section V, we show some selected numerical simulations related to the outage probability of L-branch MRC diversity receivers over i.i.d Gamma-Gamma fading channels. Finally, the paper ends with a summary of the main results.

\section{SySTEM MODEL}

The instantaneous signal-to-noise ratio (SNR) expression at the diversity receiver, in the MRC case, is given by [21]

$$
\gamma_{\text {end }}=\frac{E_{s}}{N_{0}} \sum_{\ell=1}^{L} X_{\ell},
$$

where $\frac{E_{s}}{N_{0}}$ is the SNR per symbol at the transmitter, $L$ is the number of diversity branches, and $\left\{X_{\ell}\right\}_{\ell=1}^{L}$ are the channel gains which are modeled as i.i.d Gamma-Gamma variates $\Gamma \Gamma(k, m, \Omega)$, $\ell=1, \ldots, L$, whose PDFs are given by $[8]$

$$
\begin{aligned}
f_{X_{\ell}}(x) & =\frac{2(k m)^{\frac{k+m}{2}} x^{\frac{k+m}{2}-1}}{\Gamma(m) \Gamma(k) \Omega^{\frac{k+m}{2}}} K_{k-m}\left(2\left(\frac{k m}{\Omega} x\right)^{\frac{1}{2}}\right), \\
x & \geq 0, \ell=1, \ldots, L,
\end{aligned}
$$


where $k$ and $m$ are two positive real numbers that represent the distribution parameters, $K_{\nu}(\cdot)$ is the modified Bessel function of the second kind of order $\nu, \Gamma(\cdot)$ is the Gamma function, and $\Omega$ is the mean power of the $\mathrm{RV} X_{\ell}, \ell=1, \ldots, L$.

The Gamma-Gamma distribution $\Gamma \Gamma(k, m, \Omega)$ generalizes other types of PDFs when certain values of the fading parameters are considered. For instance, it is a good approximation for the Gamma distribution when $k \rightarrow+\infty$ or $m \rightarrow+\infty$. For $m=1$ or $k=1$, it coincides with a K-distribution, while when $k=1$ and $m=1$, it reduces to the square of the double Rayleigh distribution. In small perturbations, the Gamma-Gamma model gives similar results to the log-normal model [8]. Also, since the K-distribution is a special case of the GammaGamma distribution, it can also model strong turbulence regime. To sum up, the Gamma-Gamma turbulence model allows to describe different turbulence regimes and shows a good fit with data from measurements [8].

The outage probability $P$, which quantifies the probability that the instantaneous SNR falls below a certain threshold $\gamma_{t h}$, is frequently used as a performance metric of communication systems operating over fading channels

$$
P=\mathbb{P}\left(\gamma_{e n d} \leq \gamma_{t h}\right)=\mathbb{P}\left(S_{L}=\sum_{\ell=1}^{L} X_{\ell} \leq \gamma_{0}=\frac{N_{0}}{E_{s}} \gamma_{t h}\right)
$$

At a higher level of abstraction, this amounts to determining the cumulative distribution function (CDF) of the sum of Gamma-Gamma RVs. More specifically, we are interested in the case in which the outage probability requirements are very low, i.e. of the order $10^{-9}$. This situation occurs, for instance, when studying the performance of FSO systems since they are often used for high-speed backhaul wireless links which aggregate the data generated by multiple users $[22]$.

\section{IMPORTANCE SAMPLING}

For the reader convenience, we recall first the main concepts behind IS. This will facilitate the understanding of the proposed approach that will be discussed in depth later.

Writing $P=\mathbb{E}\left[\mathbb{1}_{\left(S_{L} \leq \gamma_{0}\right)}\right]$, where $\mathbb{E}[\cdot]$ is the expectation with respect to (w.r.t) the probability measure under which the PDF of $X_{\ell}$ is $f_{X_{\ell}}(\cdot), \ell=1,2, \ldots, L$, the naive MC estimator of (3) is 
given by

$$
\hat{P}_{M C}=\frac{1}{N} \sum_{i=1}^{N} \mathbb{1}_{\left(S_{L}\left(\omega_{i}\right) \leq \gamma_{0}\right)}
$$

where $N$ is the number of MC samples, $\mathbb{1}_{(\cdot)}$ is the indicator function, and $\left\{S_{L}\left(\omega_{i}\right)\right\}_{i=1}^{N}$ are i.i.d. realizations of the RV $S_{L}$. The sequence $\left\{X_{\ell}\left(\omega_{i}\right)\right\}_{\ell=1}^{L}$ is sampled independently according to the PDFs (2), for each realization of $S_{L}$.

When the value of $P$ is very small, naive MC turns out to be computationally expensive. In fact, in this setting, a very large number of samples $N$, of the order of $100 / P$, is required to ensure that $\hat{P}_{M C}$ estimates accurately the quantity of interest with $10 \%$ relative error.

An alternative method to evaluate the probability of rare events is the IS technique [18]. In addition to reducing the computational work compared to naive MC, IS is known for its simplicity and ease of implementation compared to other variance reduction techniques. The main idea behind this method is to construct an unbiased estimator of $P$, with a smaller variance than the naive $\mathrm{MC}$ estimator, by introducing an auxiliary $\mathrm{PDF} f_{X_{\ell}}^{*}(\cdot)$. For a concise review of the use of IS in communication systems, the reader is referred to [23].

IS exploits the fact that the representation of $P$ as an expected value is not unique. In fact, we can re-write $P$ as

$$
\begin{aligned}
P & =\mathbb{E}\left[\mathbb{1}_{\left(S_{L} \leq \gamma_{0}\right)}\right]=\int_{\mathbb{R}^{L}} \mathbb{1}_{\left(S_{L} \leq \gamma_{0}\right)} \prod_{\ell=1}^{L} f_{X_{\ell}}\left(x_{\ell}\right) d x_{1} \ldots d x_{L} \\
& =\mathbb{E}^{*}\left[\mathbb{1}_{\left(S_{L} \leq \gamma_{0}\right)} \mathcal{L}\left(X_{1}, \ldots, X_{L}\right)\right]
\end{aligned}
$$

where $\mathbb{E}^{*}[\cdot]$ denotes the expectation w.r.t the probability measure under which the PDF of $X_{\ell}$ is $f_{X_{\ell}}^{*}(\cdot), \ell=1,2, \ldots, L$ and the likelihood ratio is defined as

$$
\mathcal{L}\left(X_{1}, \ldots, X_{L}\right)=\prod_{\ell=1}^{L} \frac{f_{X_{\ell}}\left(X_{\ell}\right)}{f_{X_{\ell}}^{*}\left(X_{\ell}\right)} .
$$

By defining the biased densities $\left\{f_{X_{\ell}}^{*}(\cdot)\right\}_{\ell=1}^{L}$, IS aims to "encourage" the sampling from the importance region $\left\{S_{L} \leq \gamma_{0}\right\}$. In this case, the IS estimator of (3) is

$$
\hat{P}_{I S}=\frac{1}{N^{*}} \sum_{i=1}^{N^{*}} \mathbb{1}_{\left(S_{L}\left(\omega_{i}\right) \leq \gamma_{0}\right)} \mathcal{L}\left(X_{1}\left(\omega_{i}\right), \ldots, X_{L}\left(\omega_{i}\right)\right),
$$

where for each realization $i=1, \ldots, N$, the sequence $\left\{X_{\ell}\left(\omega_{i}\right)\right\}_{\ell=1}^{L}$ are sampled independently according to the biased PDFs $\left\{f_{\ell}^{*}(\cdot)\right\}_{\ell=1}^{L}$. The main difficulty with the IS implementation lies in 
the choice of the biased PDFs $\left\{f_{X_{\ell}}^{*}(\cdot)\right\}_{\ell=1}^{L}$. A bad choice can produce a large likelihood ratio. To avoid being in such situations, many criteria have been introduced in the literature to characterize the goodness of an IS approach [24]. The bounded relative error criterion is among the most strongest ones, as it ensures, whenever achieved, the high performance of the underlying IS estimator.

Definition 1. The IS estimator has a bounded relative error if the following statement holds

$$
\limsup _{\gamma_{0} \rightarrow 0} \frac{\mathbb{E}^{*}\left[\mathbb{1}_{\left(S_{L} \leq \gamma_{0}\right)} \mathcal{L}^{2}\left(X_{1}, \ldots, X_{L}\right)\right]}{P^{2}}<+\infty .
$$

This criterion can be seen as a measure of robustness of the IS estimator. In fact, if it holds, then the number of simulation runs $N$ needed to achieve a fixed accuracy requirement remains bounded independently of how small the outage probability $P$ is. This has to be compared with naive $\mathrm{MC}$ simulation which requires the number of samples to grow as $\mathcal{O}\left(P^{-1}\right)$ in order to retrieve the same accuracy.

To quantify the efficiency of IS compared to naive MC, we introduce the following two metrics

Definition 2. The relative error of naive MC is

$$
\varepsilon=\frac{\alpha}{P} \sqrt{\frac{P(1-P)}{N}},
$$

where $\alpha=1.96$ which corresponds to a $95 \%$ confidence interval.

Similarly, we define the relative error of an IS approach as

$$
\varepsilon^{*}=\frac{\alpha}{P} \sqrt{\frac{\operatorname{Var}^{*}\left[\mathbb{1}_{\left(S_{L} \leq \gamma_{0}\right)} \mathcal{L}\left(X_{1}, \ldots, X_{L}\right)\right]}{N}},
$$

where $\operatorname{Var}^{*}[\cdot]$ is the variance w.r.t the probability measure under which the PDF of $X_{\ell}$ is $f_{X_{\ell}}^{*}(\cdot)$.

Definition 3. If we fix the relative error requirement, then, we can easily determine the number of required simulation runs. In fact, for a fixed $\epsilon_{0}$ and using Eqs. (9) and (10), the number of samples needed by naive MC simulations and IS are respectively given by

$$
\begin{aligned}
N & =(P(1-P))\left(\frac{\alpha}{P \epsilon_{0}}\right)^{2}, \\
N^{*} & =\operatorname{Var}^{*}\left[\mathbb{1}_{\left(S_{L} \leq \gamma_{0}\right)} \mathcal{L}\left(X_{1}, \ldots, X_{L}\right)\right]\left(\frac{\alpha}{P \epsilon_{0}}\right)^{2} .
\end{aligned}
$$




\section{Proposed MeAn-Shift Approach}

Our approach consists in choosing the biased PDF $\left\{f_{X_{\ell}}^{*}(\cdot)\right\}$ obtained by a mean shift of the original PDF, so that the new mean is $\Omega^{*}=\Omega-\Omega_{0}$ where $\Omega_{0}$ satisfies $0<\Omega_{0}<\Omega$ and $\Omega_{0} \rightarrow \Omega$ as $\gamma_{0} \rightarrow 0$. The biased PDF is given by

$$
\begin{aligned}
f_{X_{\ell}}^{*}(x) & =\frac{2(k m)^{\frac{k+m}{2}} x^{\frac{k+m}{2}}-1}{\Gamma(m) \Gamma(k)\left(\Omega-\Omega_{0}\right)^{\frac{k+m}{2}}} K_{k-m}\left(2\left(\frac{k m}{\Omega-\Omega_{0}} x\right)^{\frac{1}{2}}\right), \\
x & \geq 0, \ell=1, \ldots, L,
\end{aligned}
$$

The likelihood ratio (6) is then expressed as

$$
\mathcal{L}=\left(1-\frac{\Omega_{0}}{\Omega}\right)^{\frac{L}{2}(k+m)} \prod_{\ell=1}^{L} \frac{K_{k-m}\left(2\left(\frac{k m}{\Omega} X_{\ell}\right)^{\frac{1}{2}}\right)}{K_{k-m}\left(2\left(\frac{k m}{\Omega-\Omega_{0}} X_{\ell}\right)^{\frac{1}{2}}\right)} .
$$

Setting $\mathbb{E}^{*}\left[\sum_{\ell=1}^{L} X_{\ell}\right]=\gamma_{0}$, the expression of $\Omega_{0}$ is given by

$$
\Omega_{0}=\Omega-\frac{\gamma_{0}}{L}
$$

With the value of $\Omega_{0}$ at hand, we characterize in the following theorem the robustness of the proposed IS approach.

Theorem. Let $\left\{X_{\ell}\right\}_{\ell=1}^{L}$ be a sequence of i.i.d Gamma-Gamma RVs and $f_{X_{\ell}}^{*}(\cdot)$ be defined as in (13) where $\Omega_{0}$ is given by (15). Then, for $k-m>\frac{1}{2}$ such that $k-m \notin \mathbb{N}$, the IS estimator (7) has a relative bounded error.

Proof: Due to page limitation, the proof is given with details in our companion technical report in [19].

\section{Simulation Results}

In this section, the outage probability is estimated using both the naive MC as well as the proposed IS approach described in Section IV. First, the accuracy of these two methods is analyzed in both i.i.d and i.n.i.d cases. Then, the gain, in terms of required number of samples needed to meet a fixed accuracy requirement, achieved by IS w.r.t naive MC is quantified.

The behavior of the outage probability of L-branch MRC diversity receivers over i.i.d GammaGamma fading model, as a function of the SNR threshold $\gamma_{t h}$, is depicted in Fig. 1 for the number 
of branches $L \in\{4,5,6\}$. The number of simulation runs used here is $N=10^{7}$ for $\mathrm{MC}$ and $N^{*}=10^{4}$ for IS. Although there is a good agreement between MC and IS for high values of outage probabilities for each value of $L$, naive $\mathrm{MC}$, unlike IS, fails to estimate the range of low outage probabilities. For instance, for $L=4$, the naive MC estimator matches perfectly the IS estimator up to an outage probability value of the order of $10^{-5}$. Then, as we decrease the SNR threshold, the MC estimate becomes erroneous. While only $10^{4}$ simulation runs are sufficient for IS to yield a very accurate estimate of the outage probability, much more than $10^{7}$ samples must be used by naive MC in order to retrieve the same accuracy.

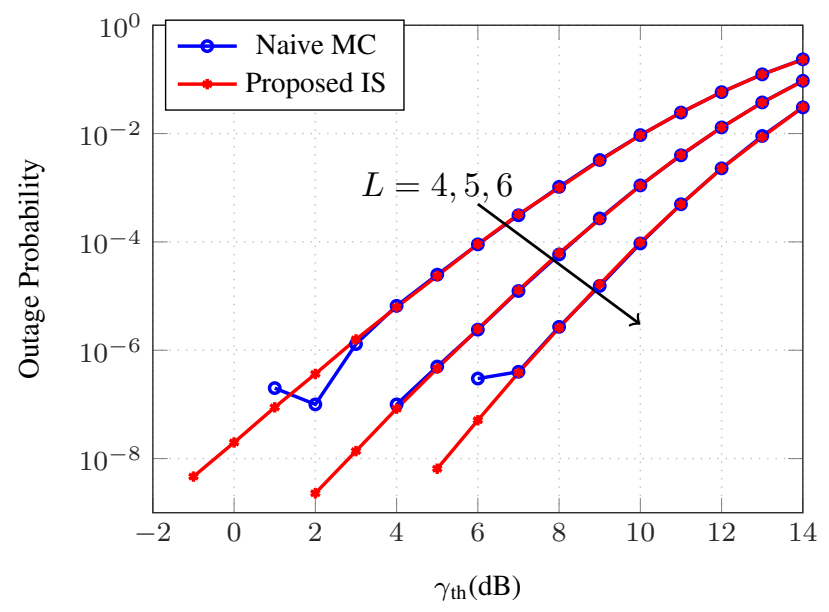

Fig. 1. Outage probability of L-branch MRC diversity receivers over i.i.d Gamma-Gamma fading model with $E_{s} / N_{0}=10$ $\mathrm{dB}, k=3.99, m=1.7$, and $\Omega=0 \mathrm{~dB}$. Number of samples $N=10^{7}$ and $N^{*}=10^{4}$.

We investigate in Fig. 2 the efficiency of our proposed IS estimator. To this end, we set both relative errors given by Definition 2 to be $\varepsilon=\varepsilon^{*}=5 \%$, and we compute, using the expressions detailed in Definition 3, the number of simulation runs $N$ and $N^{*}$ needed respectively by naive MC and IS to meet the aforementioned $95 \%$ accuracy level. From this figure, it is clearly obvious that the proposed IS method outperforms naive MC simulation in all of the three considered scenarios. Furthermore, we note that as the SNR threshold decreases, the efficiency increases. In fact, for each scenario, the number of samples $N$ is rapidly growing as the outage probability becomes smaller, whereas $N^{*}$ is almost constant over the considered range of outage probabilities. The latter statement is a consequence of the bounded relative error property of the IS estimator. For the sake of illustration, for $L=4$, the number of samples $N^{*}$ required by IS is approximately 


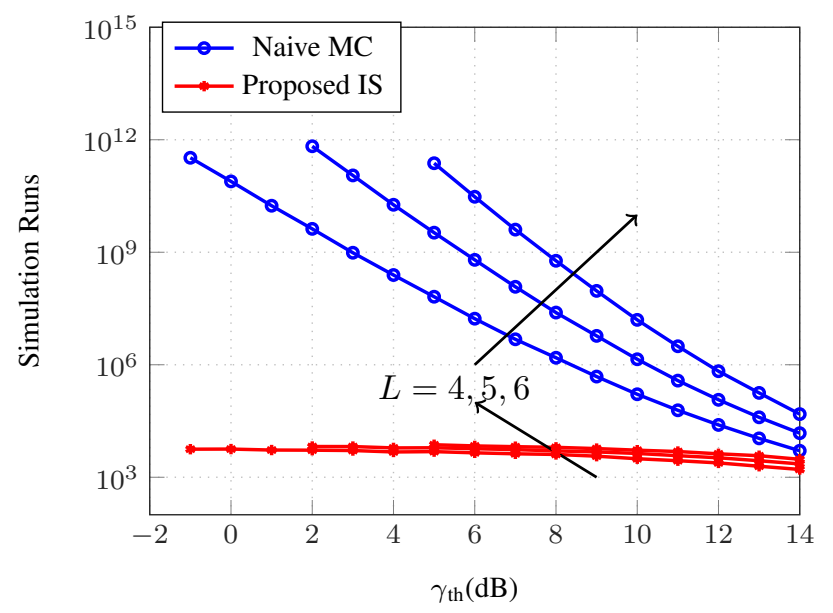

Fig. 2. Number of required simulation runs for $5 \%$ relative error for L-branch MRC diversity receivers over i.i.d Gamma-Gamma fading model with $E_{s} / N_{0}=10 \mathrm{~dB}, k=3.99, m=1.7$, and $\Omega=0 \mathrm{~dB}$.

$5 \times 10^{4}$ (respectively $6 \times 10^{7}$ ) times less than the number of samples used in MC simulations for $\gamma_{t h}=4 \mathrm{~dB}$ (respectively $\gamma_{t h}=-1 \mathrm{~dB}$ ).

\section{CONCLUSION}

In this paper, we proposed an efficient IS scheme for the estimation of the left tail of the sum i.i.d of Gamma-Gamma variates. This scheme was used to efficiently estimate the outage probability of L-branch maximum ratio combining diversity receivers over Gamma-Gamma fading channels. We showed that the proposed estimator possesses the bounded relative error. Simulation results show a significant reduction in the number of samples for the same level of accuracy which highlights the efficiency of the proposed IS estimator compared to naive MC. Due to page limitation, only the i.i.d case was discussed in this paper. In [19], we extended our results to the i.n.i.d case along with detailed proofs of the main derivations. 


\section{REFERENCES}

[1] D. J. Lewinsky, "Nonstationary probabilistic target and clutter scattering models," IEEE Transactions Antenna Propagation, vol. 31, pp. 490-498, May 1983.

[2] M. Gu and D. A. Abraham, "Using McDaniel's model to represent non-Rayleigh reverberation," IEEE Transactions Oceanic Engineering, vol. 26, pp. 348-357, July 2001.

[3] P. M. Shankar, "Error rates in generalized shadowed fading channels," Wireless Personal Communications, vol. 28, pp. 233-238, Feb. 2004.

[4] I. M. Kostic, "Analytical approach to performance analysis for channel subject to shadowing and fading," IEE Proceedings-Communications, vol. 152, pp. 821-827, Dec. 2005.

[5] P. M. Shankar, "Outage probabilities in shadowed fading channels," IEE ProceedingsCommunications, vol. 152, pp. 828-832, Dec. 2005.

[6] P. S. Bithas, N. C. Sagias, P. T. Mathiopoulos, G. K. Karagiannidis, and A. A. Rontogiannis, "On the performance analysis of digital communications over generalized-K fading channel," IEEE Commununication Letters, vol. 5, pp. 353-355, May 2006.

[7] S. Al-Ahmadi, "The Gamma-Gamma signal fading model: a survey," IEEE Antennas and Propagation Magazine, vol. 56, pp. 245-260, Oct. 2014.

[8] M. A. Al-Habash, L. C. Andrews, and R. L. Philips, "Mathematical model for the irradiance probability density function of a laser propagating yhrough turbulent media," Optical Engineering, vol. 40, pp. 1554-1562, Aug. 2001.

[9] P. Deng, M. Kavehrad, Z. Liu, Z. Zhou, and X. Yuan, "Capacity of MIMO free space optical communications using multiple partially coherent beams propagation through nonKolmogorov strong turbulence," Optics Express, vol. 21, pp. 15 213-15 229, Jul 2013.

[10] P. S. Bithas, P. T. Mathiopoulos, and S. A. Kotsopoulos, "Diversity reception over generalized-(KG) fading channels," IEEE Transactions on Wireless Communications, vol. 6, pp. 4238-4243, Dec. 2007.

[11] E. Bayaki, R. Schober, and R. K. Mallik, "Performance analysis of MIMO free-space optical systems in Gamma-Gamma fading," IEEE Transactions on Communications, vol. 57, pp. 3415-3424, Nov. 2009.

[12] S. Al-Ahmadi and H. Yanikomeroglu, "On the approximation of the generalized-K distribution by a Gamma distribution for modeling composite fading channels," IEEE 
Transactions On Wireless Communications, vol. 9, pp. 706-713, Feb. 2010.

[13] V. P. Thanh, T. Cong-Thang, and T. P. Anh, "On the MGF-based approximation of the sum of independent Gamma-Gamma random variables," in Proceedings of IEEE 81st Vehicular Technology Conference (VTC Spring), Glasgow, Scotland, May 2015, pp. 1-5.

[14] N. D. Chatzidiamantis and G. K. Karagiannidis, "On the distribution of the sum of Gamma-Gamma variates and applications in RF and optical wireless communications," IEEE Transactions on Communications, vol. 59, pp. 1298-1308, May 2011.

[15] K. P. Peppas, "A simple, accurate approximation to the sum of Gamma-Gamma variates and applications in MIMO free-space optical systems," IEEE Photonics Technology Letters, vol. 23, pp. 839-841, July 2011.

[16] J. Morio, M. Balesdent, D. Jacquemart, and C. Vergé, "A survey of rare event simulation methods for static input-output models," Simulation Modelling Practice and Theory, vol. 49, p. 287304, Nov. 2014.

[17] Y. Auffray, P. Barbillon, and J.-M. Marin, "Bounding rare event probabilities in computer experiments," Computational Statistics and Data Analysis, vol. 80, pp. 153-166, 2014.

[18] J. A. Bucklew, Introduction to Rare Event Simulation. New York, NY, USA: Springer, 2004.

[19] C. B. Issaid, N. B. Rached, A. Kammoun, M.-S. Alouini, and R. Tempone, "On the efficient simulation of the distribution of the sum of Gamma-Gamma variates with application to the outage probability evaluation over fading channels," Submitted to IEEE transactions on Communications, June 2016. [Online]. Available: http://repository.kaust.edu.sa/kaust/handle/10754/612739

[20] N. B. Rached, A. Kammoun, M.-S. Alouini, and R. Tempone, "Unified importance sampling schemes for efficient simulation of outage capacity over generalized fading channels," IEEE Journal of Selected Topics in Signal Processing, vol. 10, pp. 376-388, Mar. 2016.

[21] F. Yilmaz and M.-S. Alouini, "A unified MGF-based capacity analysis of diversity combiners over generalized fading channels," IEEE Transactions on Communications, vol. 60, pp. 862-875, Mar. 2012.

[22] Y. Li, M. Piro, and V. Angelakisi, "Design of cellular backhaul topology using the fso technology," in Proceedings of 2nd International Workshop on Optical Wireless Communications (IWOW), Newcastle, UK, Oct 2013, pp. 6-10. 
[23] P. J. Smith, M. Shafi, and H. Gao, "Quick simulation: A review of importance sampling techniques in communications systems," IEEE Journal on Selected Areas in Communications, vol. 15, p. 597613, May 1997.

[24] S. Asmussen and P. W. Glynn, Stochastic Simulation : Algorithms and Analysis, ser. Stochastic modelling and applied probability. New York: Springer, 2007. 